\section{New Japanese observatory} planned for detecting neutrinos

\section{Tokyo}

Flushed with the success of detecting neutrinos from a supernova, physicists at Tokyo University are proposing the construction of a gigantic underground observatory for neutrinos and nucleon decay that will dwarf their present facility. But a competing proposal from the same university to build a $1-\mathrm{GeV}$ proton linear accelerator may stop the super-detector project in its tracks.

In February last year, a team headed by Professor Masatoshi Kishiba of Tokyo University detected a burst of neutrinos from the supernova 1987A in the Large Magellanic Cloud during the Kamiokande-II experiment being carried out $1 \mathrm{~km}$ underground in the Kamioka mine in Gifu Prefecture (Nature 326, 121; 1987).

The Kamiokande observatory consists of a gigantic cylindrical water tank (15 m in diameter and $16 \mathrm{~m}$ deep) covered on the inside with highly sensitive photomultiplier tubes that detect Cerenkov radiation from high-energy electrons produced, for example, when neutrinos enter the tank.

The detector was originally built to observe proton decay - the grand unified theories predict that protons decay into a lepton plus meson(s) and that the leptons if charged will produce Cerenkov radiation that can be picked up by Kamiokande. But the half-life of protons has proved to be beyond the present facility's detection limit of about $10^{32}$ years

The proposed 'Super-Kamiokande' detector will have a water capacity of 45,000 tons, enough to fill more than twenty Olympic swimming pools, and will be able to observe nucleon decay on a timescale of $10^{33}$ to $10^{34}$ years, as well as to detect solar and supernova neutrinos, and, perhaps, magnetic monopoles.

Construction of the super-detector will require excavation of a gigantic cave in Kamioka mine with a volume of around $10^{5} \mathrm{~m}^{3}$ to house the cylindrical water tank which will be over $40 \mathrm{~m}$ high and $40 \mathrm{~m}$ in diameter. But this should not prove to be a major problem, according to one of the proponents of the scheme, because Mitsui Mining and Smelting Co. Ltd which owns Kamioka mine "is very cooperative to underground scientific research".

Cool crystal-clear water is readily available in the mine, and, after filtration, its average attenuation exceeds $35 \mathrm{~m}$, more than enough to allow detection of bursts of Cerenkov radiation from the centre of the gigantic tank. And calculations by $\mathrm{Dr} \mathrm{T}$. Totsuka of Tokyo University indicate that Super-Kamiokande, when armed with 11,00020 -inch photomultiplier tubes and a computer, will be able positively to identify bursts of solar neutrinos by checking that they are actually coming from the direction of the Sun.

Supporters of the scheme argue that it is becoming increasingly clear that the energies required to test the grand unified theories, which try to unify the strong, electromagnetic and weak forces, lie far beyond the capabilities of accelerators, whereas detection of proton decay with Super-Kamiokande would provide a unique opportunity to test the unification of the forces. But, ironically, it is Japan's accelerator physicists who may derail the

\section{London}

THE proposal to move the Royal Greenwich Observatory from its distinguished site at Herstmonceux Castle in Sussex to Cambridge overcame its final official hurdle last week when Cambridge City Council approved plans for the observatory's new $£ 3$-million building alongside the Institute of Astronomy. Work is expected to begin in September

The decision by the Science and Engineering Research Council (SERC) to move the Royal Greenwich Observatory (RGO), announced some 18 months ago (see Nature 321, 799; 1986), provoked considerable hostility, with SERC coming in for heavy criticism for failing to consult

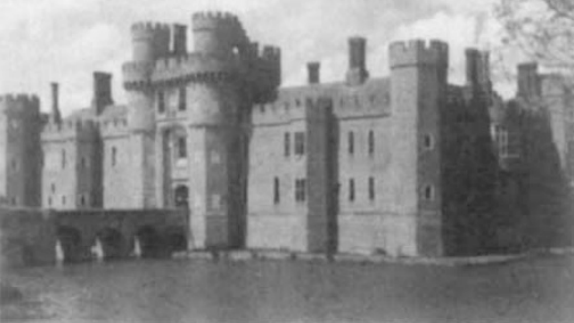

adequately with the astronomy community. The move, originally scheduled for late 1989 , now seems more likely to take place around the spring of 1990 . Delays in SERC's timetable were caused when the initial planning application for the new building in Cambridge failed to provide sufficient information about how the move would affect local job opportunities and how the 100 RGO staff would be housed.

During the past decade, Cambridge has been the focus of a proliferation of hightechnology business, dubbed the 'Cambridge phenomenon' and the city's administrators are becoming increasingly concerned that the influx of high-salaried executives is causing an imbalance in the local job market, with fewer opportunities
Super-Kamiokande project. The Institute of Nuclear Study at Tokyo University is proposing to build a $1-\mathrm{GeV}$ proton linac to be set up next to the High Energy Physics Laboratory in Tsukuba which houses the world's most powerful electron-positron collider (see Nature 331, 297; 1988).

Professor Akito Arima, vice-president of Tokyo University, says his university will have to choose later this year between the two proposals before requesting funds from the Ministry of Education, Science and Culture. He does not know which way the decision will go. But Super-Kamiokande has the advantage of being cheaper - $¥ 8,000$ million (about $\$ 60$ million) as opposed to $¥ 40,000$ million for the proton linac.

\title{
Greenwich Observatory moves a step closer to Cambridge
}

for indigenous blue-collar workers. Furthermore, property prices are being pushed beyond the means of many of the local community. The University of Cambridge has undertaken to strive to accommodate the new RGO workforce.

The initial early bitterness within RGO caused by the announcement of SERC's intentions to move the observatory has now largely subsided, although staff are still not relishing the prospect of the inevitable upheaval. Furthermore, there will be a number of redundancies, probably confined to the lower grades of staff.

The remaining imponderable is how much SERC will be able to raise from the sale of the Herstmonceux site, which will be conducted under the watchful gaze of the country's conservationists, keen that the exquisite example of a mediaeval brick-built castle, a scheduled ancient monument and grade I listed building, remains accessible to the public (it is at present open from Easter to September, and attracts some 70,000 visitors a year).

SERC says the move will cost $£ 5.5$ million, and will pay for itself. Cheaper running and operating costs at its new site are expected to save RGO some $£ 0.5$ million a year for the first five years, with the sale of the Herstmonceux site making up the balance. The site will be officially placed on the market in the spring as three lots: castle, gardens, woodland and farmland; the office complex; and the equatorial group of six telescopes (currently forming part of an astronomy exhibition). Valuations are rumoured to run as high as $£ 4$ million, although the castle market is fickle and SERC will not be drawn on the matter of price speculation.

The satellite laser ranger will remain at the Herstmonceux site, with the likelihood that SERC will lease back from the buyer the land on which the ranger sits.

Simon Hadlington 